\title{
Synthesis of trifluoromethylated dihydrofurans by addition of 1,3-dicarbonyl compounds to alkenes promoted by manganese(III) acetate
}

\author{
Emre Biçer*a and Mehmet Yılmaz ${ }^{b}$ \\ TUBITAK Marmara Research Center, Energy Institute,41470 Gebze, Kocaeli, Turkey \\ Department of Chemistry, Faculty of Arts and Sciences, Kocaeli University, 41380 Umuttepe, \\ Kocaeli, Turkey \\ E-mail:bicer_emre@yahoo.com
}

\begin{abstract}
Radical addition reaction of trifluoromethyl-1,3-dicarbonyl compounds (1a-e) with various alkenes (2a-f) was investigated in the presence of manganese(III) acetate. As a result of these reactions trifluoromethyl ketone substituted dihydrofuran and bicyclic enol ether derivatives were obtained. A formation of dihydrofuran's mechanism was proposed for all compounds. Radical addition reactions with 1,1-disubstituted alkenes were obtained in good yields, however with cyclic alkenes were shown poor yields.
\end{abstract}

Keywords: Manganese(III) acetate, dihydrofuran, oxidative addition, trifluoromethyl compounds

\section{Introduction}

The use of organofluorine compounds has been attracted significant attention due to the unique influence of a fluorine substituent on the chemical, physical and physiological properties of these compounds. Thus, organofluorine chemistry impacts many areas of everyday life and technology. ${ }^{1}$ These compounds show a large number of industrial uses in lubricants, fire extinguisher agents, surfactants, pharmaceuticals and agrochemicals. ${ }^{2}$ Since the fluorine atom is highly reactive and difficult to control, the synthesis of organic fluorine compounds is an ongoing area of research in synthetic organic chemistry. ${ }^{3}$

Traditional methods for the synthesis of organofluorine compounds are direct fluorination ${ }^{4}$ and fluoroalkylation. ${ }^{5}$ Manganese(III) mediated oxidative radical addition has become a valuable method for the formation of $\mathrm{C}-\mathrm{C}$ bonds in the last three decades. Since manganese(III) acetate is effective for the formation of C-C bonds within the intramolecular addition to form lactones, ${ }^{6}$ dihydrofurans, ${ }^{7}$ furans, ${ }^{8}$ and lactams. ${ }^{9}$ Another way of obtaining organofluorine compounds is 
addition of the corresponding fluorinated 1,3-dicarbonyl compounds with unsaturated systems mediated transition metal salts such as $\mathrm{Mn}^{3+}, \mathrm{Ce}^{4+}, \mathrm{Ag}^{+}$etc. ${ }^{10}$ Trifluoromethyl substituted dihydrofuran compounds may be achieved by using this method.

Our research group is focused on radical addition of 1,3-dicarbonyl compounds and 3oxopropanenitriles as active methylene compounds with unsaturated compounds using manganese(III) acetate and cerium(IV) ammonium nitrate, resulting in the formation of furan and dihydrofuran compounds. ${ }^{11}$ Previously, we described the synthesis of 3-trifluoroacetyl-4,5dihydrofurans and 3-[dihydrofuran-2(3H)-ylidene]-1,1,1-trifluoroacetones by the radical addition of 1,3-dicarbonyl compounds with alkenes. ${ }^{12}$ Recently, we obtained the fluoroacylated and fluoroalkylated tetrahydrobenzofurans by the treatment of trifluoromethyl-1,3-dicarbonyl compounds with various alkenes. ${ }^{13}$

In this study, we investigate the radical addition reactions of trifluoromethyl-1,3-dicarbonyl compounds (1a-e) with various alkenes (2a-f) in the presence of manganese(III) acetate resulting in the formation of trifluoromethyl ketone substituted dihydrofuran compounds.

\section{Results and Discussion}

The radical addition reactions of 2-thienyl substituted five and six-membered alkenes with trifloromethyl-1,3-dicarbonyl compounds (2a, 2b) were used and bicyclic enol ether derivatives were obtained (Table 1).

Table 1. The addition reaction of trifluoromethyl-1,3-dicarbonyl compounds with cyclic alkenes $\mathbf{2 a}$ and $\mathbf{2 b}$

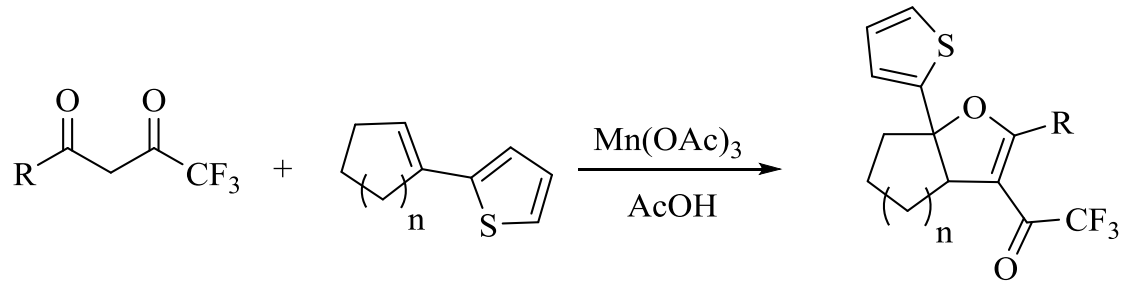

\begin{tabular}{|c|c|c|c|c|c|}
\hline & & 1a-e & $2 a-b$ & & 3a-e \\
\hline \multirow[t]{2}{*}{ Entry } & & 1,3-Dicarbonyl & \multicolumn{2}{|c|}{ Alkene } & Yield (\%) \\
\hline & & $\mathrm{R}$ & & $\mathrm{n}$ & \\
\hline 1 & $1 \mathbf{a}$ & 2-Thienyl- & $2 \mathbf{a}$ & 2 & $\mathbf{3 a}, 35$ \\
\hline 2 & $1 b$ & Phenyl- & $\mathbf{2 a}$ & 2 & $\mathbf{3 b}, 32$ \\
\hline 3 & $1 \mathbf{a}$ & 2-Thienyl- & $2 b$ & 1 & 3c, 43 \\
\hline 4 & $1 b$ & Phenyl- & $2 b$ & 1 & 3d, 39 \\
\hline 5 & $1 \mathrm{c}$ & 2-Furyl- & $2 \mathrm{~b}$ & 1 & 3e, 36 \\
\hline
\end{tabular}


The initial reactions were attempted using 4,4,4-trifluoro-1-(2-thienyl)butane-1,3-dione 1a and 4,4,4-trifluoro-1-phenylbutane-1,3-dione $\mathbf{1 b}$ with 1-(2-thienyl)-1-cyclohexen $\mathbf{2 a}$ and 1-(2thienyl)-1-cyclopentene $\mathbf{2 b}$. Trifluoromethyl-1,3-dicarbonyl compounds $\mathbf{1 a}$ and $\mathbf{1 b}$ with sixmembered alkene $\mathbf{2 a}$ were subjected to the reaction in the presence of manganese(III) acetate, 3a (35\%) and $\mathbf{3 b}(32 \%)$ were yielded in poor yields, similarly with five-membered alkene $\mathbf{2} \mathbf{b}$ gave the adduct products $\mathbf{3 c}$ and $\mathbf{3 d}$ in $43 \%$ and $39 \%$ yields, respectively. Also, the reaction of 2-furyl substituted 1,3-dicarbonyl compound $\mathbf{1 c}$ with $\mathbf{2 b}$ yielded $\mathbf{3 e}$ in $36 \%$. The characterization of the products was performed by ${ }^{1} \mathrm{H}$ - and ${ }^{13} \mathrm{C}-\mathrm{NMR}$ spectra. The signals of $\mathrm{H}$-3a protons appeared in the range of 3.59-4.05 ppm with coupling constants of ${ }^{3} J$ 6.8-9.2 Hz in 3a-e. Also, an AB system was observed with ${ }^{2} J 13.6-14.8 \mathrm{~Hz}$ was found for the diastereotopic $\mathrm{H}-4$ protons of the addition compounds 3c-e.

In order to obtain highly functionalized dihydrofuran compounds, 1,1-disubstituted alkenes (2c-f) were also used with trifluoromethyl-1,3-dicarbonyl compounds (1a-e) in the presence of manganese(III) acetate (Table 2).

Table 2. The addition reaction of trifluoromethyl-1,3-dicarbonyl compounds (1a-e) with 1,1- and 1,2-disubstituted alkenes (2c-f)

\begin{tabular}{|c|c|c|c|c|c|c|c|}
\hline \multirow{3}{*}{ Entry } & \multicolumn{2}{|r|}{ 1a-e } & \multicolumn{2}{|c|}{$2 c-f$} & \multicolumn{2}{|c|}{ 3f-o } & \\
\hline & \multicolumn{2}{|c|}{ 1,3-Dicarbonyl } & \multicolumn{4}{|c|}{ Alkene } & Yield (\%) \\
\hline & \multicolumn{3}{|c|}{$\mathrm{R}^{1}$} & $\mathrm{R}^{2}$ & $\mathrm{R}^{3}$ & $\mathrm{R}^{4}$ & \\
\hline 1 & $1 \mathbf{a}$ & 2-Thienyl- & $2 c$ & $4-\mathrm{FC}_{6} \mathrm{H}_{4-}$ & $4-\mathrm{FC}_{6} \mathrm{H}_{4}-$ & $\mathrm{H}$ & 3f, 83 \\
\hline 2 & $1 b$ & $\mathrm{Ph}-$ & $2 c$ & $4-\mathrm{FC}_{6} \mathrm{H}_{4-}$ & $4-\mathrm{FC}_{6} \mathrm{H}_{4}-$ & $\mathrm{H}$ & $\mathbf{3 g}, 78$ \\
\hline 3 & $1 c$ & 2-Furyl- & $2 c$ & $4-\mathrm{FC}_{6} \mathrm{H}_{4-}$ & $4-\mathrm{FC}_{6} \mathrm{H}_{4}-$ & $\mathrm{H}$ & 3h, 75 \\
\hline 4 & 1d & 2-Naphthyl- & $2 c$ & $4-\mathrm{FC}_{6} \mathrm{H}_{4}-$ & $4-\mathrm{FC}_{6} \mathrm{H}_{4}-$ & $\mathrm{H}$ & $3 \mathbf{i}, 73$ \\
\hline 5 & 1e & $\mathrm{CH}_{3-}$ & $2 c$ & $4-\mathrm{FC}_{6} \mathrm{H}_{4-}$ & $4-\mathrm{FC}_{6} \mathrm{H}_{4}-$ & $\mathrm{H}$ & $3 \mathbf{j}, 63$ \\
\hline 6 & 1c & 2-Furyl- & $2 d$ & $\mathrm{Ph}-$ & $\mathrm{Ph}-$ & $\mathrm{H}$ & $3 \mathbf{k}, 73$ \\
\hline 7 & 1d & 2-Naphthyl- & $2 d$ & $\mathrm{Ph}-$ & $\mathrm{Ph}-$ & $\mathrm{H}$ & 31, 69 \\
\hline 8 & 1c & 2-Furyl- & $2 \mathrm{e}$ & $\mathrm{Ph}-$ & $\mathrm{CH}_{3-}$ & $\mathrm{H}$ & $3 \mathbf{m}, 37$ \\
\hline 9 & 1d & 2-Naphthyl- & $2 \mathbf{e}$ & $\mathrm{Ph}-$ & $\mathrm{CH}_{3-}$ & $\mathrm{H}$ & $3 n, 50$ \\
\hline 10 & $1 c$ & 2-Furyl- & $2 f$ & $\mathrm{Ph}-$ & $\mathrm{Ph}-$ & $\mathrm{C}_{2} \mathrm{H}_{5^{-}}$ & 3o, 68 \\
\hline
\end{tabular}

Treatment of 1c with 4-fluorophenyl bearing alkene $\mathbf{2 c}$ and $\mathbf{2 d}$, gave the adduct products $\mathbf{3 h}$ and $\mathbf{3 k}$ in $75 \%$ and $73 \%$ yields, respectively. Similarly, the addition reactions of alkenes $\mathbf{2 c}$ and 2d with the 2-naphthyl-1,3-dicarbonyl compound 1d afforded 3i and 3l with 73\% and 69\% yields. On the other hand, when the yields of 3k-n were compared, 3k (73\%) and 31 (69\%) had 
better yields than the ones of $\mathbf{3 m}$ (37\%) and 3n (50\%) due to the phenyl group is better stabilizer than methyl. The characterization of the obtained compounds was realized by ${ }^{1} \mathrm{H}-\mathrm{NMR}$ spectra. $\mathrm{H}-4$ protons of the $\mathbf{3 f - 1}$ are appeared in the range of 3.73-4.06 ppm as singlet. But, an AB system with ${ }^{2} J_{\mathrm{AB}}$ 14.4-14.8 Hz was found for the diastereotopic $\mathrm{H}-4$ protons of $\mathbf{3 m}$ and $\mathbf{3 n}$.

As a result of these reactions, we propose a mechanism depicted in Scheme 1. According to the mechanism, while $\mathrm{Mn}^{3+}$ is reduced to $\mathrm{Mn}^{2+}$ a C-radical $\mathbf{A}$ is formed. Then, addition of $\mathbf{A}$ to alkene forms radical intermediate $\mathbf{B}$, meanwhile radical $\mathbf{B}$ is oxidized to the carbocation $\mathbf{C}$ by an equivalent amount of manganese(III) acetate. Eventually, addition of $\mathbf{C}$ can occur with two pathways $i$ and $i i$. When the reaction follows pathway $i$, product $\mathbf{E}$ is formed while with pathway ii forms product $\mathbf{G}$. Consequently, we only observed $\mathbf{E}$ and differentiated the compounds $\mathbf{E}$ and G by using ${ }^{13} \mathrm{C}-\mathrm{NMR}$.<smiles>[R]C(=O)CC(=O)C(F)(F)F</smiles><smiles>[R]C(=O)C(CC(c1ccccc1)c1ccccc1)C(=O)C(F)(F)F</smiles>

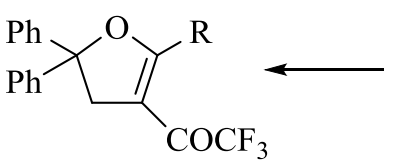

$\mathbf{E}$

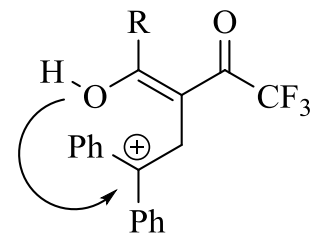

D
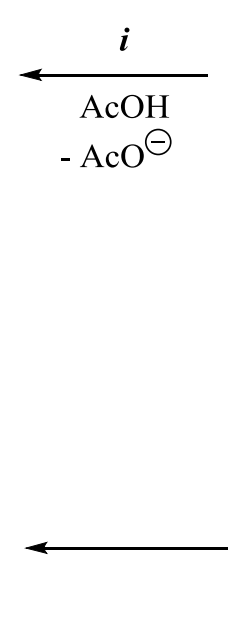

G
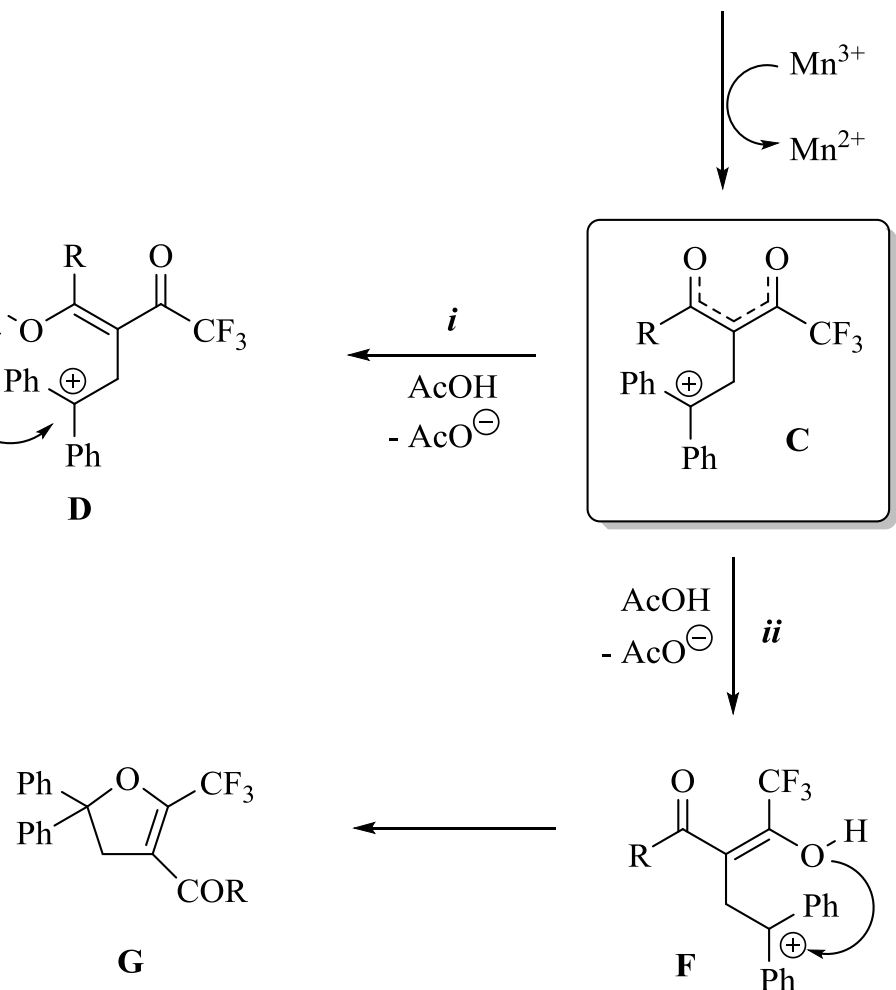

Scheme 1. Mechanism for the radical addition reaction of trifluoromethyl-1,3-dicarbonyl compounds with alkenes in the presence of manganese(III) acetate.

According to the reaction mechanism proposed in Scheme 2, it is possible to afford compounds $\mathbf{A}$ or $\mathbf{B}$ depended on the addition of 1,3-dicarbonyl compound to alkene resulting in 
the substituon of $\mathrm{CF}_{3}$ group to carbonyl carbon and $\mathrm{C} 2$ carbon of dihydrofuran. ${ }^{11 \mathrm{~m}}$ But a single product was obtained. So, the exact structure of the compound was determined by ${ }^{13} \mathrm{C}-\mathrm{NMR}$ spectra. In the spectrum of $\mathbf{3 a}$, carbonyl carbon gives a quartet at $174.8 \mathrm{ppm}\left({ }^{2} J_{\mathrm{C}-\mathrm{F}} 34.3 \mathrm{~Hz}\right)$ while the $\mathrm{C} 2$ carbon gives singlet at $166.5 \mathrm{ppm}$. If $\mathrm{CF}_{3}$ substituted to $\mathrm{C} 2$ carbon, it would appear as quartet at about $150 \mathrm{ppm}$. The reason of obtaining only single isomer $\mathbf{A}$ is due to the cyclization of the most stable form of 1,3-dicarbonyl compound ${ }^{14}$ (Scheme 1, product E). Also, it is observed that carbonyl carbon split into quartet at $173.3-176.5 \mathrm{ppm}\left({ }^{2} J_{\mathrm{C}-\mathrm{F}} 33.6-35.5 \mathrm{~Hz}\right)$ in all resulting compounds (3a-o). This shows that the $\mathrm{CF}_{3}$ is substituted to carbonyl carbon not $\mathrm{C} 2$ on the dihydrofuran.

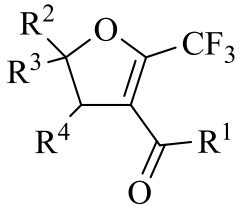

A

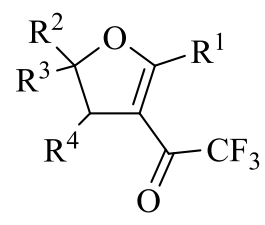

B

Scheme 2. Two possible product $\mathbf{A}$ and $\mathbf{B}$ after the radical addition of 1,3-dicarbonyl compounds 1a-e to corresponding alkenes $\mathbf{2 a - f}$.

\section{Conclusions}

In this paper, we easily obtained highly useful intermediates for the pharmaceutically important trifluoromethyl ketone containing dihydrofuran compounds. In addition, a synthetic approach in the presence of manganese(III) acetate with radical addition reaction has been investigated for the synthesis of trifluoromethyl substituted dihydrofuran compounds apart from the fluoroalkylation and direct fluorination to obtain trifluoromethyl ketone compounds.

\section{Experimental Section}

General. 4,4,4-Trifluoro-1-(2-thienyl)butane-1,3-dione 1a, 4,4,4-trifluoro-1-phenylbutane-1,3dione 1b, 4,4,4-trifluoro-1-(2-furyl)butane-1,3-dione 1c, 4,4,4-trifluoro-1-(2-naphthyl)butane1,3-dione 1d, and 1,1,1-trifluoropentane-2,4-dione 1e were purchased from Sigma-Aldrich and all were used as 1,3-dicarbonyl compounds. 1-(2-Thienyl)-1-cyclohexene 2a, 1-(2-thienyl)-1cyclopentene $\mathbf{2 b}, 4,4$ '-(ethane-1,1-diyl)bis(fluorobenzene) 2c, ethene-1,1-diyldibenzene 2d, 1,1diphenyl-1-butene $2 \mathbf{f}$ were prepared as described in the literature ${ }^{15}$ and prop-1-en-2-ylbenzene $\mathbf{2 e}$ were purchased from Sigma-Aldrich. All conjugated alkenes were freshly prepared before using in the radical additions. Manganese(III) acetate dihydrate $(98 \%)$ was prepared using an electrochemical method according to the literature. ${ }^{16}$ 
Melting points were determined using a Gallenkamp capillary melting point apparatus. IR spectra $\left(\mathrm{KBr}\right.$ disc, $\left.\mathrm{CHCl}_{3}\right)$ were obtained with a Matson 1000 FTIR spectrometer in the 400$4000 \mathrm{~cm}^{-1}$ range with $4 \mathrm{~cm}^{-1}$ resolution. ${ }^{1} \mathrm{H}-\mathrm{NMR}$ (400 MHz), ${ }^{13} \mathrm{C}-\mathrm{NMR}\left(100 \mathrm{MHz}\right.$ ), and ${ }^{19} \mathrm{~F}-$ NMR (376 MHz) spectra were recorded on a Bruker DPX-400 MHz High Performance Digital FT-NMR spectrometer in $\mathrm{CDCl}_{3}$ using TMS as internal standard. The mass spectra were measured on a Waters 2695 Alliance HPLC waters micromass 2Q (ESI method) and Micromass UK Platform II (EIMS method) spectrophotometers. Element analyses were performed on a Leco 932 CHNS-O instrument. Thin layer chromatography (TLC) was performed using Merck aluminium-packed silica gel plates. Purification of products was performed by column chromatography on silica gel (Merck silica gel 60, 40-60 $\mu \mathrm{m}$ ) or preparative TLC on silica gel of Merck $\left(\mathrm{PF}_{254-366} \mathrm{~nm}\right)$.

General procedure for the synthesis of dihydrofurans (3a-o). Manganese(III) acetate dihydrate $(0.83 \mathrm{~g}, 3 \mathrm{mmol})$ in $20 \mathrm{~mL}$ of glacial acetic acid was heated under nitrogen atmosphere to $80{ }^{\circ} \mathrm{C}$ until it dissolved. After the solution cooled down to $60{ }^{\circ} \mathrm{C}$, a solution of 1,3 -dicarbonyl compound $(2 \mathrm{mmol})$ and alkene $(1 \mathrm{mmol})$ in $5 \mathrm{~mL}$ acetic acid was added to this mixture. The reaction was completed when the initial dark brown color of the solution disappeared. Water (20 $\mathrm{mL})$ was added to this solution and extracted with $\mathrm{CHCl}_{3}(3 \times 20 \mathrm{~mL})$. The combined organic phases were neutralized with saturated $\mathrm{NaHCO}_{3}$ solution, dried over anhydrous $\mathrm{Na}_{2} \mathrm{SO}_{4}$ and evaporated. The crude products were purified by column chromatography on silica gel or preparative TLC using n-hexane/EtOAc as eluent.

1-(2,7a-Di(2-thienyl)-3a,4,5,6,7,7a-hexahydrobenzofuran-3-yl)-2,2,2-trifluoroethanone (3a). Yellow oil, 35\%, 134 mg. IR (KBr disc, $\left.\mathrm{cm}^{-1}\right)$ : $1664(\mathrm{C}=\mathrm{O}), 1531(\mathrm{C}=\mathrm{C}), 1203,1138$ (C-F), 758, 700. ${ }^{1} \mathrm{H}$ NMR $\left(400 \mathrm{MHz}, \mathrm{CDCl}_{3}\right), \delta_{\mathrm{H}}: 8.51(1 \mathrm{H}, \mathrm{dd}, J 3.6$ and $1.2 \mathrm{~Hz}), 7.69(1 \mathrm{H}, \mathrm{dd}, J 4.4$ and $1.2 \mathrm{~Hz}), 7.19(1 \mathrm{H}, \mathrm{td}, J 4.4$ and $1.2 \mathrm{~Hz}), 7.16(1 \mathrm{H}, \mathrm{dd}, J 5.2$ and $1.2 \mathrm{~Hz}), 7.04(1 \mathrm{H}, \mathrm{dd}, J 3.6$ and $1.2 \mathrm{~Hz}), 6.90(1 \mathrm{H}$, dd, $J 5.2$ and $3.6 \mathrm{~Hz}), 3.59(1 \mathrm{H}, \mathrm{t}, J 6.8 \mathrm{~Hz}, \mathrm{H} 3 \mathrm{a}), 2.60(1 \mathrm{H}, \mathrm{d}, J 15.2 \mathrm{~Hz}$, H4a), 2.26 - 2.30 (1H, m, H4b), $1.91-1.99(1 \mathrm{H}, \mathrm{m}), 1.71-1.78(2 \mathrm{H}, \mathrm{m}), 1.56-1.65(1 \mathrm{H}, \mathrm{m})$, $1.33-1.40(2 \mathrm{H}, \mathrm{m}) .{ }^{13} \mathrm{C} \mathrm{NMR}\left(100 \mathrm{MHz}, \mathrm{CDCl}_{3}\right) \delta_{\mathrm{C}}: 174.8\left(\mathrm{q},{ }^{2} J_{\mathrm{C}-\mathrm{F}} 34.3 \mathrm{~Hz}, \mathrm{C}=\mathrm{O}\right), 166.5(\mathrm{C} 2)$, 149.6, 135.7, 134.2, 131.9, 128.1, 126.7, 124.4, 123.3, 121.9 (q, $\left.{ }^{1} J_{\mathrm{C}-\mathrm{F}} 289.5 \mathrm{~Hz}, \mathrm{CF}_{3}\right), 113.2$ (C3), 90.7, 46.3, 36.6, 31.2, 21.8, 21.4. $\mathrm{m} / \mathrm{z}\left(\mathrm{ESI}^{+}\right): 385.36\left(\mathrm{MH}^{+}, 100 \%\right)$. Anal. Calcd. for $\mathrm{C}_{18} \mathrm{H}_{15} \mathrm{~F}_{3} \mathrm{O}_{2} \mathrm{~S}_{2}$ (384.44): C, 56.24; H, 3.93; S, 16.68\%. Found: C, 56.13; H, 3.85; S, $16.59 \%$.

\section{2,2,2-Trifluoro-1-(2-phenyl-7a-(2-thienyl)-3a,4,5,6,7,7a-hexahydrobenzofuran-3-}

yl)ethanone (3b). Yellow oil, 32\%, $120 \mathrm{mg}$. IR (KBr disc, $\left.\mathrm{cm}^{-1}\right)$ : 3055, 2930, 2861, 1681 (C=O), 1589 (C=C), 1199 (C-F), 1143, 758, 694. ${ }^{1} \mathrm{H}$ NMR (400 MHz, $\left.\mathrm{CDCl}_{3}\right) \delta_{\mathrm{H}}: 7.78(2 \mathrm{H}, \mathrm{dt}, J$ 7.2 and $1.6 \mathrm{~Hz}), 7.51(1 \mathrm{H}, \mathrm{tt}, J 7.2$ and $1.6 \mathrm{~Hz}), 7.42(2 \mathrm{H}, \mathrm{tt}, J 7.2$ and $1.6 \mathrm{~Hz}), 7.24(1 \mathrm{H}, \mathrm{dd}, J$ 4.8 and $1.2 \mathrm{~Hz}), 7.08(1 \mathrm{H}, \mathrm{dd}, J 3.6$ and $1.2 \mathrm{~Hz}), 6.96(1 \mathrm{H}, \mathrm{dd}, J 5.2$ and $4.0 \mathrm{~Hz}), 3.63(1 \mathrm{H}, \mathrm{td}, J$ 8.0 and $7.2 \mathrm{~Hz}, \mathrm{H} 3 \mathrm{a}), 2.54(1 \mathrm{H}, \mathrm{d}, J 14.8 \mathrm{~Hz}, \mathrm{H} 4 \mathrm{a}), 2.27-2.31(1 \mathrm{H}, \mathrm{m}, \mathrm{H} 4 \mathrm{~b}), 1.97-2.05$ (1H, m), $1.39-1.75(5 \mathrm{H}, \mathrm{m}) .{ }^{13} \mathrm{C} \mathrm{NMR}\left(100 \mathrm{MHz}, \mathrm{CDCl}_{3}\right) \delta_{\mathrm{C}}: 175.7\left(\mathrm{q},{ }^{2} J_{\mathrm{C}-\mathrm{F}} 35.1 \mathrm{~Hz}, \mathrm{C}=\mathrm{O}\right)$, 162.7(C2), 148.8, 132.1, 130.0, 127.9, 127.6, 126.6, 124.5, 123.5, 115.3 (q, ${ }^{1} J_{\mathrm{C}-\mathrm{F}} 289.5 \mathrm{~Hz}, \mathrm{CF}_{3}$ ), 114.3 (C3), 90.5, 47.1, 35.5, 29.6, 20.8, 20.7. ${ }^{19} \mathrm{~F}$ NMR (376 MHz, $\left.\mathrm{CFCl}_{3}\right) \delta_{\mathrm{F}}:-73.9\left(\mathrm{~s}, \mathrm{CF}_{3}\right)$. 
$m / z\left(\mathrm{ESI}^{+}\right): 379.4\left(\mathrm{MH}^{+}, 100 \%\right)$. Anal. Calcd. for $\mathrm{C}_{20} \mathrm{H}_{17} \mathrm{~F}_{3} \mathrm{O}_{2} \mathrm{~S}$ (378.41): C, 63.48; H, 4.53; S, 8.47\%. Found: C, 63.35; H, 4.42; S, 8.36\%.

1-(2,6a-Di(2-thienyl)-4,5,6,6a-tetrahydro-3aH-cyclopenta[b])-2,2,2-trifluoroethanone (3c). Yellow oil, 43\%, 159 mg. IR (KBr disc, $\left.\mathrm{cm}^{-1}\right)$ : $1660(\mathrm{C}=\mathrm{O}), 1531(\mathrm{C}=\mathrm{C}), 1199(\mathrm{C}-\mathrm{F}), 1140,731$, 700. ${ }^{1} \mathrm{H} \mathrm{NMR}\left(400 \mathrm{MHz}, \mathrm{CDCl}_{3}\right) \delta_{\mathrm{H}}: 8.40(1 \mathrm{H}, \mathrm{dd}, J 3.6$ and $1.2 \mathrm{~Hz}), 7.60(1 \mathrm{H}, \mathrm{dd}, J 5.2$ and 1.2 $\mathrm{Hz}), 7.20(1 \mathrm{H}, \mathrm{tt}, J 4.8$ and $1.2 \mathrm{~Hz}), 7.10(1 \mathrm{H}, \mathrm{td}, J 4.8$ and $1.2 \mathrm{~Hz}), 6.99(1 \mathrm{H}, \mathrm{dt}, J 4.0$ and 1.2 $\mathrm{Hz}), 6.91(1 \mathrm{H}, \mathrm{tt}, J 5.2$ and $1.6 \mathrm{~Hz}), 3.96(1 \mathrm{H}, \mathrm{d}, J 8.8 \mathrm{~Hz}, \mathrm{H} 3 \mathrm{a}), 2.53(1 \mathrm{H}, \mathrm{dd}, J 13.6$ and 5.2 $\mathrm{Hz}), 2.11-2.22(2 \mathrm{H}, \mathrm{m}), 1.71-1.90(3 \mathrm{H}, \mathrm{m}) .{ }^{13} \mathrm{C} \mathrm{NMR}\left(100 \mathrm{MHz}, \mathrm{CDCl}_{3}\right) \delta_{\mathrm{C}}: 174.9\left(\mathrm{q},{ }^{2} J_{\mathrm{C}-\mathrm{F}}\right.$ $34.3 \mathrm{~Hz}, \mathrm{C}=\mathrm{O}), 166.2$ (C2), 146.0, 135.6, 133.9, 130.9, 128.0, 127.2, 125.6, 123.7, 113.2 (q, ${ }^{1} J_{\mathrm{C}-\mathrm{F}}$ $289.5 \mathrm{~Hz}), 108.8$ (C3), 99.3, 54.1, 41.5, 36.5, 24.8. m/z (ESI $\left.{ }^{+}\right): 370.95\left(\mathrm{MH}^{+}, 100 \%\right)$. Anal. Calcd. for $\mathrm{C}_{17} \mathrm{H}_{13} \mathrm{~F}_{3} \mathrm{O}_{2} \mathrm{~S}_{2}$ (370.41): C, 55.12; H, 3.54; S, 17.31\%. Found: C, 55.05; H, 3.48; S, $17.27 \%$.

2,2,2-Trifluoro-1-(2-phenyl-6a-(2-thienyl)-4,5,6,6a-tetrahydro-3a $H$-cyclopenta[b]furan-3yl)ethanone (3d). Yellow oil, 39\%, $141 \mathrm{mg}$. IR (KBr disc, $\left.\mathrm{cm}^{-1}\right):$ 2963, 2870, $1681(\mathrm{C}=\mathrm{O}), 1583$ $(\mathrm{C}=\mathrm{C}), 1205(\mathrm{C}-\mathrm{F}), 1140,760,696 .{ }^{1} \mathrm{H} \mathrm{NMR}\left(400 \mathrm{MHz}, \mathrm{CDCl}_{3}\right) \delta_{\mathrm{H}}: 7.68(2 \mathrm{H}, \mathrm{dd}, J 8.0$ and 1.2 $\mathrm{Hz}), 7.42(1 \mathrm{H}, \mathrm{tt}, J 7.6$ and $1.6 \mathrm{~Hz}), 7.33(2 \mathrm{H}, \mathrm{tt}, J 8.0$ and $1.6 \mathrm{~Hz}), 7.22(1 \mathrm{H}$, dd, $J 5.2$ and 1.2 $\mathrm{Hz}), 7.02(1 \mathrm{H}, \mathrm{dd}, J 4.0$ and $1.2 \mathrm{~Hz}), 6.93(1 \mathrm{H}, \mathrm{dd}, J 5.2$ and $3.6 \mathrm{~Hz}), 3.92(1 \mathrm{H}, \mathrm{d}, J 8.8 \mathrm{~Hz}, \mathrm{H} 4)$, $2.48(1 \mathrm{H}, \mathrm{dd}, J 13.6$ and $5.6 \mathrm{~Hz}), 2.09-2.21(2 \mathrm{H}, \mathrm{m}), 1.72-1.94(3 \mathrm{H}, \mathrm{m}) .{ }^{13} \mathrm{C} \mathrm{NMR}(100 \mathrm{MHz}$, $\left.\mathrm{CDCl}_{3}\right) \delta_{\mathrm{C}}: 176.1\left(\mathrm{q},{ }^{2} J_{\mathrm{C}-\mathrm{F}} 35.1 \mathrm{~Hz}, \mathrm{C}=\mathrm{O}\right), 173.0(\mathrm{C} 2), 146.0,132.1,130.0,129.3,128.1,127.3$, 125.7, 123.7, 112.7 (q, $\left.{ }^{1} J_{\mathrm{C}-\mathrm{F}} 290.3 \mathrm{~Hz}, \mathrm{CF}_{3}\right), 110.5$ (C3), 99.5, 54.6, 41.5, 36.0, 24.8. $\mathrm{m} / \mathrm{z}\left(\mathrm{ESI}^{+}\right)$: $364.99\left(\mathrm{MH}^{+}, 100 \%\right)$. Anal. Calcd. for $\mathrm{C}_{19} \mathrm{H}_{15} \mathrm{~F}_{3} \mathrm{O}_{2} \mathrm{~S}$ (364.38): C, 62.63; H, 4.15; S, 8.80\%. Found: C, 62.55; H, 4.01; S, 8.69\%.

2,2,2-Trifluoro-1-(2-(2-furyl)-6a-(2-thienyl)-4,5,6,6a-tetrahydro-3a $H$-cyclopenta[b]furan-3yl)ethanone (3e). Yellow oil, 36\%, $127 \mathrm{mg}$. IR (KBr disc, $\left.\mathrm{cm}^{-1}\right): 3027,2925,1711(\mathrm{C}=\mathrm{O}), 1698$ $(\mathrm{C}=\mathrm{C}), 1592,1221$ (C-F), 926, 749, 693. ${ }^{1} \mathrm{H}$ NMR (400 MHz, $\left.\mathrm{CDCl}_{3}\right) \delta_{\mathrm{H}}: 8.35(1 \mathrm{H}, \mathrm{d}, J 4.0 \mathrm{~Hz})$, $7.65(1 \mathrm{H}, \mathrm{d}, J 0.8 \mathrm{~Hz}), 7.29(1 \mathrm{H}, \mathrm{dd}, J 5.2$ and $0.8 \mathrm{~Hz}), 7.11(1 \mathrm{H}, \mathrm{dd}, J 3.6$ and $0.8 \mathrm{~Hz}), 6.98(1 \mathrm{H}$, $\mathrm{dd}, J 5.2$ and $4.0 \mathrm{~Hz}), 6.61(1 \mathrm{H}, \mathrm{dd}, J 3.6$ and $1.6 \mathrm{~Hz}), 4.05(1 \mathrm{H}, \mathrm{d}, J 9.2 \mathrm{~Hz}, \mathrm{H} 3 \mathrm{a}), 2.68(1 \mathrm{H}$, dd, $J 13.6$ and $5.6 \mathrm{~Hz}), 2.21-2.32(2 \mathrm{H}, \mathrm{m}), 1.82-1.98(3 \mathrm{H}, \mathrm{m}) .{ }^{13} \mathrm{C} \mathrm{NMR}\left(100 \mathrm{MHz}, \mathrm{CDCl}_{3}\right) \delta_{\mathrm{C}}$ : 174.2 (q, $\left.{ }^{2} J_{\mathrm{C}-\mathrm{F}} 34.3 \mathrm{~Hz}, \mathrm{C}=\mathrm{O}\right), 161.3$ (C2), 146.9, 145.5, 144.2, 127.2, 125.8, 124.2, 122.6, 121.8 $\left(\mathrm{q},{ }^{1} J_{\mathrm{C}-\mathrm{F}} 289.5 \mathrm{~Hz}, \mathrm{CF}_{3}\right), 112.8,109.3(\mathrm{C} 3), 99.9,53.7,41.3,36.4,24.7 . \mathrm{m} / z\left(\mathrm{ESI}^{+}\right): 355\left(\mathrm{MH}^{+}\right.$, $100 \%$ ). Anal. Calcd. for $\mathrm{C}_{17} \mathrm{H}_{13} \mathrm{~F}_{3} \mathrm{O}_{3} \mathrm{~S}$ (354.34): C, 57.62; H, 3.70; S, 9.05\%. Found: $\mathrm{C}, 57.51 ; \mathrm{H}$, $3.59 ; \mathrm{S}, 8.98 \%$.

1-(5,5-Bis(4-fluorophenyl)-2-(2-thienyl)-4,5-dihydrofuran-3-yl)-2,2,2-trifluoroethanone (3f). Yellow oil, 83\%, $361 \mathrm{mg}$. IR ( $\mathrm{KBr}$ disc, $\mathrm{cm}^{-1}$ ): 2940, 2862, 1668 (C=O), 1569 (C=C), 1529, 1477, 1263 (C-F), 1201, 918, 850, 762, 700. ${ }^{1} \mathrm{H}$ NMR (400 MHz, CDCl 3 ) $\delta_{\mathrm{H}}: 8.66(1 \mathrm{H}, \mathrm{dd}, J 4.0$ and $1.1 \mathrm{~Hz}), 7.77(1 \mathrm{H}, \mathrm{dd}, J 5.0$ and $1.1 \mathrm{~Hz}), 7.40-7.45(4 \mathrm{H}, \mathrm{m}), 7.28(1 \mathrm{H}, \mathrm{d}, J 5.0 \mathrm{~Hz}), 7.06-$ $7.12(4 \mathrm{H}, \mathrm{m}), 3.99(2 \mathrm{H}, \mathrm{s}, \mathrm{H} 4) .{ }^{13} \mathrm{C} \mathrm{NMR}\left(100 \mathrm{MHz}, \mathrm{CDCl}_{3}\right) \delta_{\mathrm{C}}: 175.4\left(\mathrm{q},{ }^{2} J_{\mathrm{C}-\mathrm{F}} 34.6 \mathrm{~Hz}, \mathrm{C}=\mathrm{O}\right)$, 165.8 (C2), 164.7, 162.2, 139.9, 136.2, 134.8, 131.5, 129.6, 129.0, 128.3, 127.6, 119.3 (q, ${ }^{1} J_{\mathrm{C}-\mathrm{F}}$ $284 \mathrm{~Hz}, \mathrm{CF}_{3}$ ), 117.6, 117.1, 116.8, 116.5, 115.6, 115.4, 103.7 (C3), 93.1 (C5), 43.5 (C4). Anal. 
Calcd. for $\mathrm{C}_{22} \mathrm{H}_{13} \mathrm{~F}_{5} \mathrm{O}_{2} \mathrm{~S}$ (436.39): C, 60.55; H, 3.00; S, 7.35\%. Found: C, 60.44; H, 2.93; S, $7.27 \%$.

1-(5,5-Bis(4-fluorophenyl)-2-phenyl-4,5-dihydrofuran-3-yl)-2,2,2-trifluoroethanone (3g). Yellow oil, 78\%, 335 mg. IR (KBr disc, $\left.\mathrm{cm}^{-1}\right)$ : 3063, 2980, 1685 (C=O), 1575 (C=C), 1199 (C$\mathrm{F}), 1138,904,764,700 .{ }^{1} \mathrm{H} \mathrm{NMR}\left(400 \mathrm{MHz}, \mathrm{CDCl}_{3}\right) \delta_{\mathrm{H}}: 7.67(2 \mathrm{H}$, dd, J 7.2 and $1.3 \mathrm{~Hz}), 7.31-$ $7.35(1 \mathrm{H}, \mathrm{m}), 7.23-7.27(2 \mathrm{H}, \mathrm{m}), 7.16-7.21(4 \mathrm{H}, \mathrm{m}), 6.83-6.89(4 \mathrm{H}, \mathrm{m}), 3.73(2 \mathrm{H}, \mathrm{s}, \mathrm{H} 4)$.

${ }^{13} \mathrm{C}$ NMR $\left(100 \mathrm{MHz}, \mathrm{CDCl}_{3}\right) \delta_{\mathrm{C}}: 175.2\left(\mathrm{q},{ }^{2} J_{\mathrm{C}-\mathrm{F}} 35.05 \mathrm{~Hz}, \mathrm{C}=\mathrm{O}\right), 163.7,163.6,161.2$ (C2), 139.3, 133.1, 131.5, 130.4, 129.4, 128.9, 128.8, 128.6, 128.3, 128.1, 127.8, 127.4, 127.3, 126.8, 121.1 (q, ${ }^{1} J_{\mathrm{C}-\mathrm{F}} 291.7 \mathrm{~Hz}, \mathrm{CF}_{3}$ ), 116.7, 116.6, 104.8 (C3), 92.7 (C5), 44.7 (C4). ${ }^{19} \mathrm{~F}-\mathrm{NMR}(376$ $\left.\mathrm{MHz}, \mathrm{CFCl}_{3}\right) \delta_{\mathrm{F}}:-75.92\left(\mathrm{~s}, \mathrm{CF}_{3}\right),-113.00$ (s, C-F). Anal. Calcd. for $\mathrm{C}_{24} \mathrm{H}_{15} \mathrm{~F}_{5} \mathrm{O}_{2}$ (430.37): C, $66.98 ; \mathrm{H}, 3.51 \%$. Found: C, 66.86; H, 3.44\%.

1-(5,5-Bis(4-fluorophenyl)-2-(2-furyl)-4,5-dihydrofuran-3-yl)-2,2,2-trifluoroethanone (3h). Pale yellow oil, 75\%, $315 \mathrm{mg}$. IR (KBr disc, $\left.\mathrm{cm}^{-1}\right)$ : 3028, 2922, $1685(\mathrm{C}=\mathrm{O}), 1585,1556(\mathrm{C}=\mathrm{C})$, 1209 (C-F), 1136, 758, 702. ${ }^{1} \mathrm{H}$ NMR $\left(400 \mathrm{MHz}, \mathrm{CDCl}_{3}\right) \delta_{\mathrm{H}}: 8.42(1 \mathrm{H}, \mathrm{d}, J 3.7 \mathrm{~Hz}), 7.74(1 \mathrm{H}, \mathrm{d}$, $J 1.3 \mathrm{~Hz}), 7.47(4 \mathrm{H}, \mathrm{dd}, J 7.0$ and $2.1 \mathrm{~Hz}), 7.12(4 \mathrm{H}, \mathrm{t}, J 8.6 \mathrm{~Hz}), 6.68(1 \mathrm{H}, \mathrm{dd}, J 3.7 \mathrm{and} 1.7 \mathrm{~Hz})$, $3.96(2 \mathrm{H}, \mathrm{s}, \mathrm{H} 4) .{ }^{13} \mathrm{C} \mathrm{NMR}\left(100 \mathrm{MHz}, \mathrm{CDCl}_{3}\right) \delta_{\mathrm{C}}: 173.4$ (q, $\left.{ }^{2} J_{\mathrm{C}-\mathrm{F}} 35.5 \mathrm{~Hz}, \mathrm{C}=\mathrm{O}\right), 163.7$ (C2), 161.2, 160.1, 146.8, 143.9, 139.1, 139.0, 127.6, 122.6, 118.5 (q, ${ }^{1} J_{\mathrm{C}-\mathrm{F}} 290.3 \mathrm{~Hz}, \mathrm{CF}_{3}$ ), 115.7 , 115.6, 115.3, 112.8, 103.3 (C3), 93.1 (C5), 42.8 (C4). $\left.{ }^{19} \mathrm{~F} \mathrm{NMR} \mathrm{(376} \mathrm{MHz,} \mathrm{CFCl}_{3}\right) \delta_{\mathrm{F}}:-76.05$ (s, $\mathrm{CF}_{3}$ ), -113.52 (s, C-F). Anal. Calcd. for $\mathrm{C}_{22} \mathrm{H}_{13} \mathrm{~F}_{5} \mathrm{O}_{3}$ (420.33): C, 62.86; H, 3.12\%. Found: C, $62.79 ; \mathrm{H}, 3.01 \%$.

\section{1-(5,5-Bis(4-fluorophenyl)-2-(2-naphthyl)-4,5-dihydrofuran-3-yl)-2,2,2-trifluoroethanone}

(3i). Pale yellow oil, 73\%, $350 \mathrm{mg}$. IR ( $\mathrm{KBr}$ disc, $\left.\mathrm{cm}^{-1}\right): 3055,2930,2861,1681$ (C=O), 1589 $(\mathrm{C}=\mathrm{C}), 1544,1448,1276,1199(\mathrm{C}-\mathrm{F}), 1143,758,694 .{ }^{1} \mathrm{H} \mathrm{NMR}\left(400 \mathrm{MHz}, \mathrm{CDCl}_{3}\right) \delta_{\mathrm{H}}: 8.58$ $(1 \mathrm{H}, \mathrm{s}), 7.99(1 \mathrm{H}, \mathrm{d}, J 7.8 \mathrm{~Hz}), 7.83-7.92(3 \mathrm{H}, \mathrm{m}), 7.64(1 \mathrm{H}, \mathrm{td}, J 7.0$ and $1.6 \mathrm{~Hz}), 7.59(1 \mathrm{H}, \mathrm{td}$, $J 7.0$ and $1.6 \mathrm{~Hz}), 7.45-7.50(4 \mathrm{H}, \mathrm{m}), 7.09-7.15(4 \mathrm{H}, \mathrm{m}), 4.05(2 \mathrm{H}, \mathrm{s}, \mathrm{H} 4) .{ }^{13} \mathrm{C}$ NMR $(100$ $\left.\mathrm{MHz}, \mathrm{CDCl}_{3}\right) \delta_{\mathrm{C}}: 175.4\left(\mathrm{q},{ }^{2} J_{\mathrm{C}-\mathrm{F}} 34.3 \mathrm{~Hz}, \mathrm{C}=\mathrm{O}\right), 163.9(\mathrm{C} 2), 161.5,139.7,135.3,132.8,132.7$, $131.5,131.4,129.5,128.6,128.0,127.9,127.8,127.0,126.1,125.7,118.6\left(\mathrm{q},{ }^{1} J_{\mathrm{C}-\mathrm{F}} 290.3 \mathrm{~Hz}\right.$, $\left.\mathrm{CF}_{3}\right), 116.0,115.9,115.8,115.7,105.2$ (C3), 93.0 (C5), 43.7 (C4). ${ }^{19} \mathrm{~F} \mathrm{NMR}\left(376 \mathrm{MHz}, \mathrm{CFCl}_{3}\right)$ $\delta_{\mathrm{F}}:-76.198\left(\mathrm{~s}, \mathrm{CF}_{3}\right),-113.8$ (s, C-F). Anal. Calcd. for $\mathrm{C}_{28} \mathrm{H}_{17} \mathrm{~F}_{5} \mathrm{O}_{2}$ (480.43): C, 70.00; H, 3.57\%. Found: C, 69.95; H, 3.49\%.

1-(5,5-Bis(4-fluorophenyl)-2-methyl-4,5-dihydrofuran-3-yl)-2,2,2-trifluoroethanone (3j). Pale yellow oil, 63\%, $231 \mathrm{mg}$. IR (KBr disc, $\left.\mathrm{cm}^{-1}\right)$ : 3063, 2980, 1685 (C=O), 1575 (C=C), 1199 (C-F), 1138, 904, 764, 700. ${ }^{1} \mathrm{H}$ NMR (400 MHz, $\left.\mathrm{CDCl}_{3}\right) \delta_{\mathrm{H}}: 7.37(4 \mathrm{H}, \mathrm{dd}, J 8.8$ and $2.1 \mathrm{~Hz})$, $7.07(4 \mathrm{H}, \mathrm{dd}, J 8.7$ and $2.1 \mathrm{~Hz}), 3.81(2 \mathrm{H}, \mathrm{s}, \mathrm{H} 4), 2.52(3 \mathrm{H}, \mathrm{s}) .{ }^{13} \mathrm{C} \mathrm{NMR}\left(100 \mathrm{MHz}, \mathrm{CDCl}_{3}\right) \delta_{\mathrm{C}}$ : 176.5 (q, $\left.{ }^{2} J_{\mathrm{C}-\mathrm{F}} 34.9 \mathrm{~Hz}, \mathrm{C}=\mathrm{O}\right), 175.7$ (C2), 163.6, 161.2, 139.8, 139.6, 139.5, 139.2, 127.9, 127.8, 127.7, 127.5, 121.0 (q, $\left.{ }^{1} J_{\mathrm{C}-\mathrm{F}} 289.5 \mathrm{~Hz}, \mathrm{CF}_{3}\right), 105.5$ (C4), 93.7 (C5), $42.4(\mathrm{C} 4), 15.7\left(\mathrm{CH}_{3}\right)$. Anal. Calcd. for $\mathrm{C}_{19} \mathrm{H}_{13} \mathrm{~F}_{5} \mathrm{O}_{2}$ (368.30): C, 61.96; H, 3.56\%. Found: C, 61.85; H, 3.48\%.

2,2,2-Trifluoro-1-(2-(2-furyl)-5,5-diphenyl-4,5-dihydrofuran-3-yl)ethanone (3k). Yellow oil, 73\%, 280 mg. IR (KBr disc, $\left.\mathrm{cm}^{-1}\right)$ : 3028, 2922, $1685(\mathrm{C}=\mathrm{O}), 1585,1556(\mathrm{C}=\mathrm{C}), 1209$ (C-F), 1136, 758, 692. ${ }^{1} \mathrm{H}$ NMR $\left(400 \mathrm{MHz}, \mathrm{CDCl}_{3}\right) \delta_{\mathrm{H}}: 8.41(1 \mathrm{H}, \mathrm{d}, J 4.0 \mathrm{~Hz}), 7.72(1 \mathrm{H}, \mathrm{d}, J 1.6 \mathrm{~Hz})$, 
$7.29-7.49(10 \mathrm{H}, \mathrm{m}), 6.65(1 \mathrm{H}, \mathrm{dd}, J 3.2$ and $1.6 \mathrm{~Hz}), 4.01(2 \mathrm{H}, \mathrm{s}, \mathrm{H} 4) .{ }^{13} \mathrm{C} \mathrm{NMR}(100 \mathrm{MHz}$, $\left.\mathrm{CDCl}_{3}\right) \delta_{\mathrm{C}}: 173.8\left(\mathrm{q},{ }^{2} J_{\mathrm{C}-\mathrm{F}} 34.3 \mathrm{~Hz}, \mathrm{C}=\mathrm{O}\right), 160.5(\mathrm{C} 2), 146.7,144.2,143.5,128.6,128.1,125.7$, 122.4, 118.6, (q, $\left.{ }^{1} J_{\mathrm{C}-\mathrm{F}} 289.6 \mathrm{~Hz}, \mathrm{CF}_{3}\right)$ 112.7, 103.5 (C3), 93.9 (C5), 43.2 (C4). m/z (\%): 384 $\left(6.35, \mathrm{M}^{+}\right), 366\left(4.68, \mathrm{M}^{+}-\mathrm{H}_{2} \mathrm{O}\right), 355\left(0.22, \mathrm{M}^{+}-\mathrm{C}_{2} \mathrm{H}_{5}\right), 315\left(3.59, \mathrm{M}^{+}-\mathrm{CF}_{3}\right), 287\left(5.31, \mathrm{M}^{+}-\right.$ $\mathrm{CF}_{3} \mathrm{CO}$ ), 77 (32.23, $\mathrm{C}_{6} \mathrm{H}_{5}{ }^{+}$). Anal. Calcd. for $\mathrm{C}_{22} \mathrm{H}_{15} \mathrm{~F}_{3} \mathrm{O}_{3}$ (384.35): C, 68.75; H, 3.93\%. Found: C, 68.67; H, 3.84\%.

2,2,2-Trifluoro-1-(2-naphthyl)-5,5-diphenyl-4,5-dihydrofuran-3-yl)ethanone (31). Yellow oil, 69\%, 306 mg. IR (KBr disc, $\left.\mathrm{cm}^{-1}\right)$ : 3055, 2930, 2861, $1681(\mathrm{C}=\mathrm{O}), 1589$ (C=C), 1544, 1448, 1276, 1199 (C-F), 1143, 758, 694. ${ }^{1} \mathrm{H}$ NMR (400 MHz, $\left.\mathrm{CDCl}_{3}\right) \delta_{\mathrm{H}}: 8.56(1 \mathrm{H}, \mathrm{s}), 7.78-7.94$ $(6 \mathrm{H}, \mathrm{m}), 7.29-7.54(6 \mathrm{H}, \mathrm{m}), 7.29-7.40(5 \mathrm{H}, \mathrm{m}), 4.06(2 \mathrm{H}, \mathrm{m}, \mathrm{H} 4) .{ }^{13} \mathrm{C} \mathrm{NMR}(100 \mathrm{MHz}$, $\left.\mathrm{CDCl}_{3}\right) \delta_{\mathrm{C}}: 175.5\left(\mathrm{q},{ }^{2} J_{\mathrm{C}-\mathrm{F}} 34.3 \mathrm{~Hz}, \mathrm{C}=\mathrm{O}\right), 171.9(\mathrm{C} 2), 143.8,137.6,135.0,132.4,132.3,131.1$, 130.0, 129.2, 128.7, 128.3, 128.2, 128.1, 127.7, 127.6, 126.6, 121.3 (q, ${ }^{1} J_{\mathrm{C}-\mathrm{F}} 289.6 \mathrm{~Hz}, \mathrm{CF}_{3}$ ), 105.1 (C3), 93.6 (C5), 43.3 (C4). ${ }^{19} \mathrm{~F}$ NMR (376 MHz, $\left.\mathrm{CFCl}_{3}\right) \delta_{\mathrm{F}}:-76.148\left(\mathrm{~s}, \mathrm{CF}_{3}\right) . \mathrm{m} / z\left(\mathrm{ESI}^{+}\right)$: $445\left(\mathrm{MH}^{+}, 100 \%\right)$. Anal. Calcd. for $\mathrm{C}_{28} \mathrm{H}_{19} \mathrm{~F}_{3} \mathrm{O}_{2}$ (444.44): C, 75.67; H, 4.31\%. Found: C, 75.61; $\mathrm{H}, 4.28 \%$.

2,2,2-Trifluoro-1-(2-(2-furyl)-5-methyl-5-phenyl-4,5-dihydrofuran-3-yl)ethanone (3m). Yellow oil, 37\%, $119 \mathrm{mg}$. IR ( $\mathrm{KBr}$ disc, $\left.\mathrm{cm}^{-1}\right)$ : $1671(\mathrm{C}=\mathrm{O}), 1539(\mathrm{C}=\mathrm{C}), 1207(\mathrm{C}-\mathrm{F}), 1136 .{ }^{1} \mathrm{H}$ NMR $\left(400 \mathrm{MHz}, \mathrm{CDCl}_{3}\right) \delta_{\mathrm{H}}: 8.43(1 \mathrm{H}, \mathrm{d}, J 3.6 \mathrm{~Hz}), 7.70(1 \mathrm{H}, \mathrm{d}, J 1.2 \mathrm{~Hz}), 7.26-7.47(5 \mathrm{H}, \mathrm{m})$, $6.40(1 \mathrm{H}, \mathrm{dd}, J 3.6$ and $1.2 \mathrm{~Hz}), 3.66(1 \mathrm{H}, \mathrm{d}, J 14.8 \mathrm{~Hz}, \mathrm{H} 4 \mathrm{a}), 3.50(1 \mathrm{H}, \mathrm{d}, J 14.8 \mathrm{~Hz}, \mathrm{H} 4 \mathrm{~b}), 1.87$

$(3 \mathrm{H}, \mathrm{s}) .{ }^{13} \mathrm{C}$ NMR $\left(100 \mathrm{MHz}, \mathrm{CDCl}_{3}\right) \delta_{\mathrm{C}}: 173.3$ (q, $\left.{ }^{2} J_{\mathrm{C}-\mathrm{F}} 34.3 \mathrm{~Hz}, \mathrm{C}=\mathrm{O}\right), 161.0(\mathrm{C} 2), 146.8$, 144.9, 144.6, 128.9, 128.1, 124.4, 122.4, 118.8 (d, $\left.{ }^{1} J_{\mathrm{C}-\mathrm{F}} 289.6 \mathrm{~Hz}, \mathrm{CF}_{3}\right), 112.9,103.5(\mathrm{C} 3), 91.4$ (C5), 43.4 (C4), $29.3\left(\mathrm{CH}_{3}\right) . \mathrm{m} / z(\%): 322\left(\mathrm{M}^{+}, 10.30\right), 304\left(\mathrm{M}^{+}-\mathrm{H}_{2} \mathrm{O}, 5.45\right), 253\left(\mathrm{M}^{+}-\mathrm{CF}_{3}\right.$, 4.06), $91\left(\mathrm{C}_{6} \mathrm{H}_{5} \mathrm{CH}_{2}^{+}, 13.05\right), 77\left(\mathrm{C}_{6} \mathrm{H}_{5}{ }^{+}\right.$, 52.06). Anal. Calcd. for $\mathrm{C}_{17} \mathrm{H}_{13} \mathrm{~F}_{3} \mathrm{O}_{3}$ (322.28): $\mathrm{C}$, 63.36; H, 4.07\%. Found: C, 63.25; H, 4.00\%.

2,2,2-Trifluoro-1-(5-methyl-2-(2-naphthyl)-5-phenyl-4,5-dihydrofuran-3-yl)ethanone (3n). Pale yellow oil, 50\%, $191 \mathrm{mg}$. IR (KBr disc, $\left.\mathrm{cm}^{-1}\right)$ : 3065, 2967, 2930, 1660 (C=O), $1585(\mathrm{C}=\mathrm{C})$, 1548, $1197(\mathrm{C}-\mathrm{F}), 1141,756,696 .{ }^{1} \mathrm{H}$ NMR (400 MHz, $\left.\mathrm{CDCl}_{3}\right) \delta_{\mathrm{H}}: 8.53(1 \mathrm{H}, \mathrm{s}), 7.96(2 \mathrm{H}, \mathrm{d}, J$ $8.0 \mathrm{~Hz}), 7.89(2 \mathrm{H}, \mathrm{dd}, J 7.2$ and $2.0 \mathrm{~Hz}), 7.59(1 \mathrm{H}, \mathrm{td}, J 8.0$ and $1.6 \mathrm{~Hz}), 7.56(1 \mathrm{H}, \mathrm{td}, J 8.4$ and $1.6 \mathrm{~Hz}), 7.34-7.49(5 \mathrm{H}, \mathrm{m}), 3.63(1 \mathrm{H}, \mathrm{dd}, J 14.4$ and $1.2 \mathrm{~Hz}, \mathrm{H} 4 \mathrm{a}), 3.52(1 \mathrm{H}, \mathrm{dd}, J 14.4$ and 1.2 $\mathrm{Hz}, \mathrm{H} 4 \mathrm{~b}), 1.90(3 \mathrm{H}, \mathrm{s}) .{ }^{13} \mathrm{C} \mathrm{NMR}\left(100 \mathrm{MHz}, \mathrm{CDCl}_{3}\right) \delta_{\mathrm{C}}: 175.5$ (q, $\left.{ }^{2} J_{\mathrm{C}-\mathrm{F}} 33.6 \mathrm{~Hz}, \mathrm{C}=\mathrm{O}\right), 172.6$ (C2), 144.9, 135.0, 132.3, 130.9, 129.3, 128.8, 128.2, 127.9, 127.7, 127.6, 126.7, 125.6, 124.2, 118.5 (q, $\left.{ }^{1} J_{\mathrm{C}-\mathrm{F}} 289.5 \mathrm{~Hz}, \mathrm{CF}_{3}\right) 104.7(\mathrm{C} 3), 90.8(\mathrm{C} 5), 43.8(\mathrm{C} 4), 29.2\left(\mathrm{CH}_{3}\right) .{ }^{19} \mathrm{~F}$ NMR $(376 \mathrm{MHz}$, $\left.\mathrm{CFCl}_{3}\right) \delta_{\mathrm{F}}:-76.342\left(\mathrm{~s}, \mathrm{CF}_{3}\right) . \mathrm{m} / z\left(\mathrm{ESI}^{+}\right): 383\left(\mathrm{MH}^{+}, 100 \%\right)$. Anal. Calcd. for $\mathrm{C}_{23} \mathrm{H}_{17} \mathrm{~F}_{3} \mathrm{O}_{2}$ (382.38): C, 72.24; H, 4.48\%. Found: C, 72.15; H, $4.41 \%$.

1-(4-Ethyl-2-(2-furyl)-5,5-diphenyl-4,5-dihydrofuran-3-yl)-2,2,2-trifluoroethanone (3o). Dark yellow oil, 68\%, 280 mg. IR (KBr disc, $\left.\mathrm{cm}^{-1}\right)$ : 3059, 2965, 2930, $1646(\mathrm{C}=\mathrm{O}), 1606(\mathrm{C}=\mathrm{C})$, 1211, $1134(\mathrm{C}-\mathrm{F}) .{ }^{1} \mathrm{H}$ NMR $\left(400 \mathrm{MHz}, \mathrm{CDCl}_{3}\right) \delta_{\mathrm{H}}: 8.21(1 \mathrm{H}, \mathrm{d}, J 4.0 \mathrm{~Hz}), 7.71(1 \mathrm{H}, \mathrm{d}, J 1.6 \mathrm{~Hz})$, $7.65(2 \mathrm{H}, \mathrm{d}, J 7.6 \mathrm{~Hz}), 7.46(2 \mathrm{H}, \mathrm{d}, J 7.6 \mathrm{~Hz}), 7.26-7.35(6 \mathrm{H}, \mathrm{m}), 6.62(1 \mathrm{H}, \mathrm{d}, J 3.2 \mathrm{~Hz}), 4.15$ $(1 \mathrm{H}, \mathrm{t}, J 5.6 \mathrm{~Hz}, \mathrm{H} 4), 1.44-1.62(2 \mathrm{H}, \mathrm{m}), 0.54(3 \mathrm{H}, \mathrm{t}, J 7.6 \mathrm{~Hz}) .{ }^{13} \mathrm{C} \mathrm{NMR}\left(100 \mathrm{MHz}, \mathrm{CDCl}_{3}\right)$ $\delta_{\mathrm{C}}: 174.1\left(\mathrm{q},{ }^{2} J_{\mathrm{C}-\mathrm{F}} 35.1 \mathrm{~Hz}, \mathrm{C}=\mathrm{O}\right), 159.6(\mathrm{C} 2), 146.8,144.6,143.6,139.5,128.4,128.1,127.6$, 
126.5, 125.9, 122.2, 118.6 (q, ${ }^{1} J_{\mathrm{C}-\mathrm{F}} 289.5 \mathrm{~Hz}, \mathrm{CF}_{3}$ ), 112.8, 112.6, 111.3 (C3), 97.1 (C5), 49.0 (C4), 25.4, 10.3. $\mathrm{m} / z\left(\mathrm{ESI}^{+}\right): 413\left(\mathrm{MH}^{+}, 100 \%\right)$. Anal. Calcd. for $\mathrm{C}_{24} \mathrm{H}_{19} \mathrm{~F}_{3} \mathrm{O}_{3}$ (412.40): $\mathrm{C}, 69.90$; H, 4.64\%. Found: C, 69.82; H, 4.57\%.

\section{Acknowledgements}

The authors are grateful to Kocaeli University BAP (2008/28, 2010/57 and 2012/28) Science Research Foundations for financial support. The author Emre Biçer would like to thank to Mrs. Hilal Biçer for her encouragement, support and endless patience and help for evaluation of NMR data during the typing of this paper.

\section{References}

1. (a) Lemal, D. M. J. Org. Chem. 2004, 69, 1. http://dx.doi.org/10.1021/jo0302556 PMid:14703372

(b) Thayer, A. M. Chemical and Engineering News 2006, 84, 15. http://dx.doi.org/10.1021/cen-v084n033.p015

2. (a) Uneyama, K. Organofluorine Chemistry; Blackwell: Oxford, 2006. http://dx.doi.org/10.1002/9780470988589

(b) Kirsch, P. Modern Fluoroorganic Chemistry; Wiley-VCH: Weinheim, Germany, 2004. http://dx.doi.org/10.1002/352760393X

(c) Chambers, R. D. Fluorine in Organic Chemistry; Blackwell: Oxford, 2004.

(d) Hiyama, T. Organofluorine Compounds. Chemistry and Application; SpringerVerlag: Berlin, 2000.

3. (a) Tomashenko, O. A.; Grushin, V. V. Chem. Rev. 2011, 111, 4475. http://dx.doi.org/10.1021/cr1004293 PMid:21456523

(b) Kitazume, T.; Yamazaki, T. Experimental Methods in Organic Fluorine Chemistry; Gordon and Breach Science: Amsterdam, 1998.

(c) Hudlicky, M.; Pavlath, A. E. Chemistry of Organic Fluorine Compounds II; American Chemical Society: Washington DC, 1995.

4. (a) Brooke, G. M.; J. Fluorine Chem. 1997, 86, 1.

http://dx.doi.org/10.1016/S0022-1139(97)00006-7

(b) Brace, N. O. J. Fluorine Chem. 1999, 93, 1. http://dx.doi.org/10.1016/S0022-1139(98)00255-3

5. (a) Wilkinson, J. A. Chem. Rev. 1992, 92, 505. http://dx.doi.org/10.1021/cr00012a002

(b) McClinton, M. A.; McClinton D. A. Tetrahedron 1992, 48, 6555. http://dx.doi.org/10.1016/S0040-4020(01)80011-9 
(c) Shi, G. Q.; Xu, Y. Y.; Xu, M. Tetrahedron 1991, 47, 1629.

http://dx.doi.org/10.1016/S0040-4020(01)96907-8

6. (a) Corey, E. J.; Kang, M.-C. J. Am. Chem. Soc. 1984, 106, 5384.

http://dx.doi.org/10.1021/ja00330a076

(b) Fristad, W. E.; Peterson, J. R. J. Org. Chem. 1985, 50, 10.

http://dx.doi.org/10.1021/jo00201a003

7. (a) Mellor, J. M.; Mohammed, S. Tetrahedron Lett. 1991, 32, 7111.

http://dx.doi.org/10.1016/0040-4039(91)85054-9

(b) Mellor, J. M.; Mohammed, S. Tetrahedron 1993, 49, 7547.

http://dx.doi.org/10.1016/S0040-4020(01)87229-X

(c) Nishino, H.; Nguyen, V.; Yoshinaga, S.; Kurosawa, K. J. Org. Chem. 1996, 61, 8264.

http://dx.doi.org/10.1021/jo960939w

(d) Fujino, R.; Nishino, H. Synthesis 2005, 731.

(e) Snider, B. B.; Kiselgof, J. Y.; Foxman, B. M. J. Org. Chem. 1998, 63, 7945.

http://dx.doi.org/10.1021/jo981238x

(f) Curry, L.; Hallside, M. S.; Powell, L. H.; Sprague, S. J.; Burton, J. W. Tetrahedron 2009, $65,10882$.

http://dx.doi.org/10.1016/j.tet.2009.09.112

(g) Snider, B. B.; Han, L. and Xie, C. J. Org. Chem. 1997, 62, 6978.

http://dx.doi.org/10.1021/jo9708506

(h) Demir, A. S.; Emrullahoğlu, M. Curr. Org. Synth. 2007, 4, 223.

http://dx.doi.org/10.2174/157017907780598871

(i) Snider, B. B. Chem. Rev. 1996, 96, 339.

http://dx.doi.org/10.1021/cr950026m PMid:11848756

(j) Ali, M. F.; Çalışkan, R.; Şahin, E.; Balci, M. Tetrahedron 2009, 65, 1430.

http://dx.doi.org/10.1016/j.tet.2008.12.012

(k) Çalışkan, R.; Ali, M. F.; Şahin, E.; Watson, W. H.; Balci, M. J. Org. Chem. 2007, 72, 3353.

http://dx.doi.org/10.1021/jo0625711 PMid:17385919

(1) Çalışkan, R.; Pekel, T.; Watson, W. H.; Balci, M. Tetrahedron Lett. 2005, 46, 6227.

http://dx.doi.org/10.1016/j.tetlet.2005.07.051

(m) Südemen, M. B.; Zengin, M.; Genç, H. and Balci, M. Turk. J. Chem. 2011, 35, 1.

8. (a) Melikyan, G. G.; Sargsyan, A. B. and Badanyan, S. O. Chem. Heterocycl. Compd. 1989, 606.

http://dx.doi.org/10.1007/BF00470014

(b) Gregory, B.; Parsons, A. F.; Thomas, C. B. Tetrahedron 2001, 57, 4719.

http://dx.doi.org/10.1016/S0040-4020(01)00375-1

9. (a) Cabri, W.; Candiani, I.; Bedeschi, A. Tetrahedron Lett. 1992, 33, 4783.

http://dx.doi.org/10.1016/S0040-4039(00)61285-6 
(b) Antenni, B.; Cerreti, A.; D'Annibale, A.; Resta, S.; Trogolo, C. Tetrahedron 1998, 54, 12029.

10. (a) Wang, Y.; Zhu, S. Tetrahedron 2001, 57, 3383.

http://dx.doi.org/10.1016/S0040-4020(01)00183-1

(b) Leconte, S.; Ruzziconi, R. J. Fluorine Chem. 2002, 117, 167.

http://dx.doi.org/10.1016/S0022-1139(02)00161-6

11. (a) Y1lmaz, M.; Pekel, A. T. Synth. Commun. 2001, 31, 2189.

http://dx.doi.org/10.1081/SCC-100104471

(b) Y1lmaz, M.; Pekel, A. T. Synth. Commun. 2001, 31, 3871.

http://dx.doi.org/10.1081/SCC-100108239

(c) Yılmaz, M.; Biçer, E. and Pekel, A. T. Turk. J. Chem. 2005, 29, 579.

(d) Yılmaz, M.; Uzunalioğlu, N. and Pekel, A. T. Tetrahedron 2005, 61, 8860.

http://dx.doi.org/10.1016/j.tet.2005.07.019

(e) Alagoz, O.; Y1lmaz, M. and Pekel, A. T. Synth. Commun. 2006, 36, 1005.

http://dx.doi.org/10.1080/00397910500501516

(f) Burgaz, E. V.; Y1lmaz, M.; Pekel, A. T. and Öktemer, A. Tetrahedron 2007, 63, 7229.

http://dx.doi.org/10.1016/j.tet.2007.04.088

(g) Y1lmaz, M.; Yakut, M. and Pekel, A. T. Synth. Commun. 2008, 38, 914.

http://dx.doi.org/10.1080/00397910701845456

(h) Yılmaz, M.; Uzunalioğlu, N.; Yakut, M.; Pekel, A. T. Turk. J. Chem. 2008, 32, 411.

Y1lmaz, E. V. B.; Y1lmaz, M.; Öktemer, A. Arkivoc 2011, 363.

(i) Y1lmaz, M. Helv. Chim. Acta 2011, 94, 1335.

http://dx.doi.org/10.1002/hlca.201000440

(j) Loğoğlu, E.; Yılmaz, M.; Katircioğlu, H.; Yakut, M.; Mercan, S. Med. Chem. Res. 2010, $19,490$.

http://dx.doi.org/10.1007/s00044-009-9206-8

(1) Biçer, E.; Yılmaz, M.; Karataş, M.; Pekel, A. T. Helv. Chim. Acta 2012, 95, 795.

http://dx.doi.org/10.1002/hlca.201100397

(m) Y1lmaz, M. Tetrahedron 2011, 67, 8255.

http://dx.doi.org/10.1016/j.tet.2011.08.098

(n) Biçer, E.; Yılmaz, M.; Burgaz, E. V.; Pekel, A. T. Helv. Chim. Acta 2013, 96, 135.

http://dx.doi.org/10.1002/hlca.201200098

12. Y1lmaz, M.; Pekel, A. T. J. Fluorine Chem. 2005, 126, 401.

http://dx.doi.org/10.1016/j.jfluchem.2005.02.002

13. Y1lmaz, M.; Pekel, A. T. J. Fluorine Chem. 2011, 132, 628.

http://dx.doi.org/10.1016/j.jfluchem.2011.06.023

14. Sloop, J. C.; Bumgardner, C. L.; Washington, G.; Loehle, W. D.; Sankar, S. S.; Lewis, A. B. J. Fluorine Chem. 2006, 127, 780.

http://dx.doi.org/10.1016/j.jfluchem.2006.02.012 
15. (a) Yuan, D.-Y.; Tu, Y.-Q. and Fan, C.-A. J. Org. Chem. 2008, 73, 7797.

http://dx.doi.org/10.1021/jo801434b PMid:18720969

(b) Su, W.; Urgaonkar, S.; McLaughlin, P. A. and Verkade, J. G. J. Am. Chem. Soc. 2004, 126, 16433.

http://dx.doi.org/10.1021/ja0450096 PMid:15600345

(c) Namai, H.; Ikeda, H.; Kato, N. and Mizuno, K. J. Phys. Chem. A 2007, 111, 4436.

http://dx.doi.org/10.1021/jp0683081 PMid:17472352

(d) Xi, Z.; Liu, B. and Chen, W. J. Org. Chem. 2008, 73, 3954.

http://dx.doi.org/10.1021/jo800197u PMid:18412386

(e) Serijan, K. T. and Wise, P. H. J. Am. Chem. Soc. 1952, 74, 365.

http://dx.doi.org/10.1021/ja01122a022

16. Y1lmaz, M.; Y1lmaz, E. V. B.; Pekel, A. T. Helv. Chim. Acta 2011, 94, 2027.

http://dx.doi.org/10.1002/hlca.201100105 